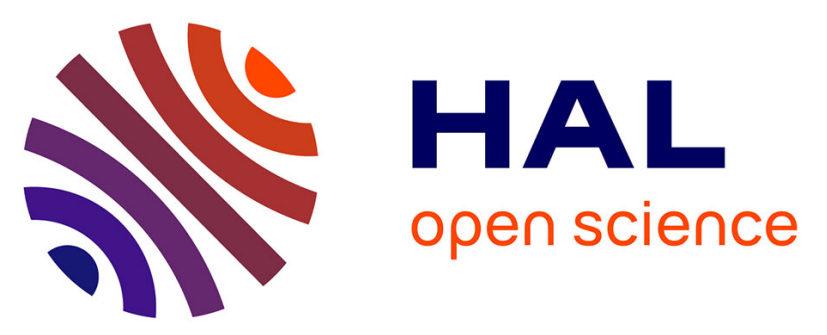

\title{
Increased overall survival independent of RECIST response in metastatic breast cancer patients continuing trastuzumab treatment: evidence from a retrospective study
}

Manuela Campiglio, Rosaria Bufalino, Marco Sandri, Elisa Ferri, Rosa Anna Aiello, Andrea Matteis, Marcella Mottolese, Sabino Placido, Patrizia Querzoli, Antonio Jirillo, et al.

\section{- To cite this version:}

Manuela Campiglio, Rosaria Bufalino, Marco Sandri, Elisa Ferri, Rosa Anna Aiello, et al.. Increased overall survival independent of RECIST response in metastatic breast cancer patients continuing trastuzumab treatment: evidence from a retrospective study. Breast Cancer Research and Treatment, 2011, 128 (1), pp.147-154. 10.1007/s10549-011-1484-4 . hal-00630792

\section{HAL Id: hal-00630792 https://hal.science/hal-00630792}

Submitted on 11 Oct 2011

HAL is a multi-disciplinary open access archive for the deposit and dissemination of scientific research documents, whether they are published or not. The documents may come from teaching and research institutions in France or abroad, or from public or private research centers.
L'archive ouverte pluridisciplinaire HAL, est destinée au dépôt et à la diffusion de documents scientifiques de niveau recherche, publiés ou non, émanant des établissements d'enseignement et de recherche français ou étrangers, des laboratoires publics ou privés. 


\section{Increased overall survival independent of RECIST response in metastatic breast cancer}

\section{patients continuing trastuzumab treatment: evidence from a retrospective study}

${ }^{1}$ Manuela Campiglio, ${ }^{1}$ Rosaria Bufalino, ${ }^{1}$ Marco Sandri, ${ }^{1}$ Elisa Ferri, ${ }^{2}$ Rosa Anna Aiello, ${ }^{3}$ Andrea

De Matteis, ${ }^{4}$ Marcella Mottolese, ${ }^{5}$ Sabino De Placido, ${ }^{6}$ Patrizia Querzoli, ${ }^{7}$ Antonio Jirillo, ${ }^{8}$ Alberto Bottini, ${ }^{9}$ Manuela Fantini, ${ }^{10}$ Andrea Bonetti, ${ }^{11}$ Fulvia Pedani, ${ }^{12}$ Maria Mauri, ${ }^{13}$ Annamaria Molino, ${ }^{14}$ Antonella Ferro, ${ }^{1}$ Serenella M. Pupa, ${ }^{1}$ Marianna Sasso, ${ }^{1}$ Sylvie Ménard**, ${ }^{16}$ Andrea Balsari and ${ }^{1}$ Elda Tagliabue.

** On behalf of the Demetra group

A. Balsari and E. Tagliabue contributed equally as senior authors.

${ }^{* *}{ }^{2}$ Eleonora Taibi, ${ }^{2}$ Giuseppe Nuciforo, ${ }^{3}$ Gianfranco Di Feo, ${ }^{4}$ Giulio Metro, ${ }^{4}$ Federica Cuppone, ${ }^{5}$ Giuseppe Cancello, ${ }^{6}$ Maria Lunardi, ${ }^{7}$ Annamaria Scola, ${ }^{8}$ Alessandra Bersiga, ${ }^{8}$ Simone Bonardi, ${ }^{9}$ Lorenzo Gianni, ${ }^{9}$ Alberto Ravaioli, ${ }^{10}$ Marta Zaninelli, ${ }^{10}$ Laura Furini, ${ }^{11}$ Ester Berardengo, ${ }^{12}$ Leopoldo Costarelli, ${ }^{13}$ Eliana Gilioli, ${ }^{14}$ Michela Frisinghelli, ${ }^{15}$ Alberto Morabito, ${ }^{17}$ Mario Brandi, ${ }^{17}$ Giovanni Simone, ${ }^{18}$ Giovanna Cavazzini, ${ }^{18}$ Alberto Bellomi, ${ }^{19}$ Giorgio Mustacchi, ${ }^{19}$ Fabrizio Zanconati, ${ }^{20}$ Alessandro Bertolini, ${ }^{20}$ Salvatore Ambrosi, ${ }^{21}$ Angelo Di Leo, ${ }^{21}$ Claudino Wederson, ${ }^{22}$ Anna Sapino, ${ }^{22}$ Isabella Castellano, ${ }^{23}$ Dario Giuffrida, ${ }^{23}$ Lorenzo Memeo, ${ }^{24}$ Andrea Decensi.

${ }^{1}$ Fondazione IRCCS Istituto Nazionale dei Tumori, via Venezian 1, 20133, Milan; ${ }^{2}$ Humanitas Centro Catanese di Oncologia, Catania; ${ }^{3}$ Istituto Nazionale Tumori, Pascale, Napoli; ${ }^{4}$ Istituto Tumori Regina Elena, Roma; ${ }^{5}$ Università Federico II, Napoli; ${ }^{6}$ Università di Ferrara; ${ }^{7}$ Oncologia Medica 2, Istituto Oncologico Veneto IRCCS, Padova; ${ }^{8}$ Azienda Istituti Ospedalieri Cremona;

${ }^{9}$ Ospedali degli Infermi, Azianda USL-Rimini; ${ }^{10}$ Ospedale Mater Salutis, Legnano; ${ }^{11}$ Ospedale 
San Giovanni, Torino ${ }^{12}$ Ospedale San Giovanni-Addolorata, Roma; ${ }^{13}$ Università di Verona; ${ }^{14}$ Ospedale Santa Chiara, Trento; ${ }^{15}$ Istituto di Statistica Medica e Biometria, Università degli Studi, Milano; ${ }^{16}$ Dipartimento di Morfologia Umana e Scienze Biomediche "Città Studi", Università degli Studi di Milano; ${ }^{17}$ IRCCS-Ospedale Oncologico, Bari; ${ }^{18}$ Azienda Ospedaliera C. Poma, Mantova; ${ }^{19}$ A. S.S. Centro Oncologico, Trieste; ${ }^{20}$ Ospedale Civile di Sondrio, Sondrio;

${ }^{21}$ Ospedale Misericordia e Dolce, Prato; ${ }^{22}$ Azienda Ospedaliera Molinette, Torino; ${ }^{23}$ Istituto Oncologico del Mediterraneo, Viagrande; ${ }^{24}$ E.O. Ospedali Galliera, Genova.

*Corresponding author: Sylvie Ménard, Fondazione IRCCS Istituto Nazionale Tumori, phone: +39-02-23902564; fax: +39-0223902692; e-mail: sylvie.menard@ @istitutotumori.mi.it 


\section{ABSTRACT}

\section{Purpose}

Recent studies have reported the potential clinical utility for metastatic breast cancer (MBC) patients of continuing trastuzumab beyond progression. Based on those results, here we have examined the benefits of trastuzumab-continuation by specifically evaluating RECIST responses upon first line trastuzumab-treatment as a potential predictive marker for therapeutic effect of trastuzumab-continuation beyond metastatic disease progression.

\section{Methods}

We carried out a retrospective analysis of 272 HER2 positive MBC patients under trastuzumab treatment at 22 different oncology Italian centers during the years of 2000 and 2001 who progressed under first line trastuzumab-treatment. The primary end point of the study was the survival from the date of first documented progression upon first line trastuzumab treatment of disease. Data analysis involved the use of matching on propensity score to balance variables between treated and untreated subjects and to reduce bias.

\section{Results}

Of the 272 HER2-positive MBC patients, 154 (56.6\%) continued treatment. 79 (51.3\%) of those 154 patients showed responses based on RECIST criteria during first-line trastuzumab-treatment. Of the 118 patients that suspended trastuzumab, RECIST responses had been observed in 44 (37.3\%). Cox proportional hazards analysis of progressed patients, matched using propensity score, showed that discontinuation of trastuzumab at metastatic disease progression was a risk factor for significantly reduced overall survival in both responder $(\mathrm{HR}=2.23 ; 95 \% \mathrm{CI}=1.03$ to 4.82) and non-responder groups $(\mathrm{HR}=3.53,95 \% \mathrm{CI}=1.73$ to 7.21 ), with no significant differences in the two estimated HRs ( $\mathrm{p}$-value of the likelihood-ratio test $=0.690$ ). 


\section{Conclusions}

Continued trastuzumab treatment after disease progression has clinically and statistically significant effects in both RECIST responder and non-responder MBC patients.

Keywords: HER2, trastuzumab, metastatic breast cancer, RECIST, propensity score matching 


\section{INTRODUCTION}

First line treatment of HER2 positive metastatic breast cancer (MBC) patients with the antiHER2 monoclonal antibody trastuzumab is now well established [1, 2]. However, patients may develop resistance to trastuzumab and show metastatic disease progression. Several retrospective studies published thus far (reviewed in $[3,4]$ ) $[5,6]$ have indicated a certain overall response rate, clinical benefit and extended time to progression from the use of a second trastuzumabcontaining regimen in HER2-positive MBC patients who progressed on first-line trastuzumab treatment. Moreover, two recent randomized trials designed to directly evaluate the efficacy of continuing trastuzumab after progression demonstrated the potential clinical utility of continuing trastuzumab even for patients with progressive disease $[7,8]$. To further evaluate the benefits of trastuzumab-continuation therapy we carried out a retrospective analysis of 272 HER2 positive MBC patients first line trastuzumab treated in the metastatic setting at different oncology centers in Italy, who continued or discontinued trastuzumab at progression. Since RECIST (Response Evaluation Criteria in Solid Tumors) was used for determining efficacy of trastuzumabcontaining regimens by the oncologists participating in this study, we specifically evaluated RECIST responses upon first line trastuzumab treatment, as a potential predictive marker for the therapeutic effects of trastuzumab-continuation beyond metastatic disease progression. The present study is the first report of increased overall survival independent of RECIST response achievement on initial chemotherapy regimens that include trastuzumab. 


\section{METHODS}

\section{Patients}

272 patients with HER2-positive MBC all receiving first line trastuzumab in the metastatic setting during 2000-2001 in 22 Italian oncologic centers and recorded as in progression under trastuzumab treatment during the 2000-2007 observation period were considered for this study. Following progression, 154 patients continued trastuzumab-containing regimens, whereas 118 patients stopped trastuzumab and changed therapy. All primary tumors were scored 3+ by immunohistochemistry (Herceptest) or 2+/FISH-positive prior to first line trastuzumab treatment for MBC.

\section{Response evaluation}

Clinical response of patients during first line trastuzumab treatment was evaluated according to RECIST 1.0 criteria [9] for the target lesions as follows: i) complete response (CR) based on disappearance of all lesions; ii) partial response (PR) based on at least $30 \%$ reduction of the sum of maximum lesion diameters; iii) progressive disease (PD) based on at least $20 \%$ increase in the sum of maximum lesion diameters; and iv) stable disease (SD) based on a variation of the sum of maximum target lesion diameters between PR and PD. Clinical response of non-target lesions was categorized as: complete response (CR), progression (PD) or noCR/noPD. Response to trastuzumab was evaluated based on ultrasound, total body scan, magnetic resonance imaging, computed axial tomography or other radiological examination. Patients were considered as responsive when they presented a complete or partial response in at least one metastatic lesion. Independent central response review has been done at Fondazione IRCCS-Istituto Nazionale Tumori. The institutional review board of each participant institution approved the observational study. 


\section{Statistical methods}

To reduce potential bias in effects of treatment choices by physicians/patients or of differences in prognosis before treatment, either of which might result in large differences between treated and untreated groups in covariates, we used a propensity score [10] to assess the conditional probability of treatment according to the individual's covariates and to balance treatment choicerelated variables such that the analysis simulates random assignment. We estimated a propensity score using a logistic regression model where the outcome variable is the oncologists' decision to continue/discontinue trastuzumab and the explanatory variables are the covariates selected according to their variable importance, estimated using random forests [11]. Our random forest contained 30000 trees and 6 random features to split each node. Mean decrease in prediction accuracy was the estimated measure of variable importance.

Multivariate 1:1 matching with replacement performed by a 0.1 caliper matching on the estimated propensity score, together with an exact matching with site of metastases, generated $238+238$ matched treated and controls units; 34 (12.5\%) observations were dropped by matching. The degree of covariate imbalance was measured using the standardized (mean and proportion) difference proposed by Austin et al [12].

Overall Survival (OS) of trastuzumab-treated patients was calculated as the time from the date of first documented progression of disease until death from any cause or until the end of the observation period. The hazard ratios related to trastuzumab treatment suspension after disease progression were estimated on matched data by a Cox proportional hazards model with random effects (frailty), stratified by investigational center [13]. Predictive covariates for survival were selected according to their variable importance, estimated using survival random forests [14]. The assumption of proportional hazards was verified using the test of Grambsch and Therneau 
[15], based on Schoenfeld residuals. The proportional hazards assumption was tested globally and for each covariate, with all null hypotheses set at a 5\% level of significance.

Differences of treatment effects in the responder and non-responder subgroups were investigated considering in the Cox model the interaction between patient response to trastuzumab-based therapy and continuation/discontinuation of treatment and by testing (using a likelihood-ratio test) whether the coefficient of the interaction term was significantly different from zero. The survival curve of Figure 1 was estimated on unmatched data by the Kaplan-Meier method. Figure 2 shows the predicted survivor functions estimated on matched data using the Cox proportional hazard model with frailty.

Missing values of the analyzed covariates were imputed by the method described in Iacus and Porro [16] based on proximity of random forests. Imputed data were used only for variable selection and propensity score estimation. Matching and model estimation were applied to selected variables with missing values. The Cox proportional hazard model was estimated using 442 observations ( 34 were deleted due to missingness).

Statistical analyses were carried out using the R software for statistical computing, v. 2.8.1 ( $R$ Development Core Team, 2008), with the following packages: randomForest, randomSurvivalForest, survival and Matching.

\section{RESULTS}

To evaluate RECIST responses upon first line trastuzumab treatment, as a potential predictive marker for any therapeutic effect of trastuzumab-continuation beyond metastatic disease progression, we have examined the benefits of trastuzumab-continuation therapy by carrying out 
a retrospective analysis of 272 HER2 positive MBC patients under treatment at 22 different oncology centers in Italy during the years of 2000 and 2001 (Fig. 1). Our analysis included 154 (56.6\%) progressed patients who continued trastuzumab for an additional 1 month to 5.5 years after progression (median exposure time to trastuzumab of 15.5 months), and 118 patients that were changed to a different chemotherapeutic regimen. Of the 154 patients that continued trastuzumab, 79 (51.3\%) showed responses (CR+PR) based on RECIST criteria during first-line trastuzumab treatment. Of the 118 patients that suspended trastuzumab, RECIST responses had been observed in $44(37.3 \%)$. The clinical-pathological characteristics of these patients are shown in Table 1.

Continuation of trastuzumab led to a significant increase in overall survival $(\mathrm{p}<0.001)$ in both RECIST responder (Fig. 2a) and non-responder groups (Fig. 2b). Specifically, RECIST responder patients continuing trastuzumab showed a median survival of 27 months $(95 \% \mathrm{CI}=24$ to 37$)$ compared to 12 months $(95 \% \mathrm{CI}=7$ to 16$)$ for those halting trastuzumab $(\mathrm{p}<0.001)$. Similarly, within RECIST non-responder patients the median survival benefit was $21(95 \% \mathrm{CI}=$ 18 to 38 ) versus 11 months $(95 \% \mathrm{CI}=8$ to $15, \mathrm{p}<0.001)$. The strong gain in overall survival in patients non-responsive to first line trastuzumab treatment who continued trastuzumab at disease progression was unexpected and surprising. It is possible that the survival benefit was due to clinical-pathological differences between those patients selected to continue trastuzumab therapy versus those that were switched to a different regimen. The continuation group represented patients of younger age (52 vs. 56 years), smaller size of first metastatic lesion at progression (20 vs. $25 \mathrm{~mm})$, a higher number of resected lymph nodes (18 vs. 15$)$, more taxane $(68.8 \%$ vs. $39.8 \%)$ and gemcitabine (14.3\% vs. $0.8 \%)$ therapy, fewer visceral-only metastases $(8.4 \%$ vs.21.2\%), more multiple metastases ( $80.5 \%$ vs. $59.3 \%)$ and fewer liver-only metastases $(6.5 \%$ 
vs. $16.9 \%$ ), a higher number of target lesions (2 vs. 1), and a higher response to trastuzumabbased therapy (51.3\% vs. $37.3 \%)$ in the metastatic disease (see also Supplementary Table 1).

To reduce bias due to patient selection, we used the method of propensity score matching estimated by a logistic regression model using continuation/discontinuation of trastuzumab as the outcome variable and the following covariates as predictors: taxane treatment, number of resected lymph nodes, axillary dissection, tumor size, response to trastuzumab-based therapy, multiple metastases, gemcitabine, time to progression, target and non-target metastases, age, site of metastases, aromatase inhibitor, number of positive lymph nodes and number of target lesions. These variables were selected using the graph of the estimated variable importances in the Supplementary Figure 1. The area under the ROC curve of the logistic model was 0.80 (95\% CI $=0.75$ to 0.86 ). The estimate values of the model's parameters are given in Supplementary Table 2.

The most predictive variables of survival were: response to trastuzumab therapy, size of first metastatic lesion at progression, menopause, brain metastases, site of metastases, other drugs associated to trastuzumab therapy, time to progression, aromatase inhibitor associated to trastuzumab therapy, and HER2 score (see the estimated variable importance in the Supplementary Figure 2). Propensity score 1:1 matching with replacement generated 238 matched treated and control patients. The two groups reached a "good" balance (standardized mean differences below 10) for all the variables associated with continuation/discontinuation of trastuzumab treatment and for predictors of survival (see Supplementary Table 1). Limiting the analysis to the matched patients (Fig. 3) still showed that discontinuation of trastuzumab at metastatic disease progression was a risk factor that significantly reduced overall survival in both responder $(\mathrm{HR}=2.23 ; 95 \% \mathrm{CI}=1.03$ to 4.82$)$ and non-responder groups $(\mathrm{HR}=3.53,95 \% \mathrm{CI}=$ 
1.73 to 7.21 ), with no significant differences in the two estimated HRs (p-value of the likelihoodratio test $=0.690$ ). These results suggest that continued trastuzumab treatment after disease progression has clinically and statistically significant effects in both responder and nonresponder subgroups, even after balancing the differences in the groups who continued or discontinued the treatment. In addition, responders and non-responders did not show significant differences in overall survival $(\mathrm{HR}=0.91,95 \% \mathrm{CI}=0.46$ to $1.67, \mathrm{p}=0.760)$.

\section{DISCUSSION}

Two recent randomized clinical trials have documented the benefit on overall response of continued use of trastuzumab in women with HER2 positive breast cancer who experienced progression during trastuzumab treatment $[7,8]$. Here we show from a study of clinical practice in Italy that continued trastuzumab treatment after disease progression has clinically and statistically significant effects, remarkably in both RECIST responder and non-responder MBC patients upon first line trastuzumab treatment.

Although we are aware that retrospective studies suffer from limitations, such as the heterogeneity we found in the patients either continuing or halting trastuzumab beyond progression, we reduced bias by building a predicted survival function on matched patients balanced for variables associated with continuation/discontinuation of trastuzumab treatment and for predictors of survival. Finding a significant clinical benefit in both RECIST responder and non-responder patients continuing trastuzumab at progression in Cox proportional hazards analysis of progressed patients, matched using propensity score, strengthened our results. Specifically, in our sample the overall survival approximately doubled in patients with RECIST 
responses continuing trastuzumab compared to those halting trastuzumab (median overall survival 27 versus 12 months). More interestingly, a similar significant increase (21 versus 11 months) was found also in non-responder patients.

While a number of retrospective analyses have shown a potential advantage for the "continuation policy" (reviewed in [3-6]) in agreement with our results, two retrospective studies indicated no statistically significant benefit from continuing trastuzumab $[17,6]$. In these studies, carried out by the same group, the authors themselves suggest that patients continuing trastuzumab might have been subject to closer clinical evaluation because of the weekly administration schedule of the antibody compared to the control arm of untreated patients, and an earlier detection of disease progression may have partially clouded the benefit of continuation.

To explain the gain in survival beyond progression in RECIST non-responder patients first observed in our series, we speculate that the two major mechanisms of action described for trastuzumab i.e., antibody-dependent cell-mediated cytotoxicity (ADCC) and cytostatic activity through the blockage of HER2 proliferation pathways [18] can explain their effectiveness in responder and non-responder patients, alike. ADCC likely accounts for tumor shrinkage and is thus probably the main mechanism reflected by the RECIST system response evaluation. ADCCmediated tumor destruction requires a functional innate immune system [19] presumably compromised in metastatic patients who have received one or more chemotherapy regimens. However, for those patients lacking cellular components that mediate ADCC and that do not account for RECIST responses, trastuzumab therapy might still be beneficial because of its cytostatic activity: inhibition of HER2-mediated proliferation pathways could lead to improved survival even without a decrease in the tumor mass.

With the exclusion of patients with tumors insensitive to HER2 blockage, including those with 
PTEN or p27 loss [20,21], or expressing a truncated HER2 receptor [22], or displaying enhanced Akt signaling, or signaling through other receptors such as cMet [23] and integrins [24], or those producing high amount of growth factors leading to increased activation of HER2 no longer sensitive to antibody blockage [25] continuation of trastuzumab treatment in patients who show no RECIST response may lead to a clinically relevant cytostatic response. Compared with cytotoxic chemotherapy, trastuzumab-based therapy and other similar targeted therapies often result in disease stabilization or delayed disease progression rather than tumor shrinkage. Thus, to predict benefits of trastuzumab continuation at progression calculated in terms of survival beyond progression, indicators other than RECIST should be considered. Promising results obtained in preclinical models have shown that molecular imaging techniques such as PET may be adequate to detect trastuzumab-induced inhibition of HER2+ tumor growth [26], but clinical explorations in randomized trials are warranted.

In conclusion, our findings suggests that continued trastuzumab treatment after disease progression has clinically and statistically significant effects in both RECIST responder and nonresponder $\mathrm{MBC}$, indicating that continuing trastuzumab beyond disease progression has efficacy also in the latter group. Our results may also suggest that is possible to restart treatment with trastuzumab even in patients relapsed in the adjuvant setting.

\section{ACKNOWLEDGEMENTS}

This work was supported by AIRC (Associazione Italiana per la Ricerca sul Cancro) and FIRB/MUR (Ministero Università e Ricerca) and Roche spa.

\section{REFERENCES}

1. Slamon DJ, Leyland-Jones B, Shak S, Fuchs H, Paton V, Bajamonde A, Fleming T, Eiermann W, Wolter J, 
Pegram M, Baselga J, Norton L (2001) Use of chemotherapy plus a monoclonal antibody against HER2 for metastatic breast cancer that overexpresses HER2. N Engl J Med 344:783-792.

2. Marty M, Cognetti F, Maraninchi D, Snyder R, Mauriac L, Tubiana-Hulin M, Chan S, Grimes D, Anton A, Lluch A, Kennedy J, O'Byrne K, Conte P, Green M, Ward C, Mayne K, Extra JM (2005) Randomized phase II trial of the efficacy and safety of trastuzumab combined with docetaxel in patients with human epidermal growth factor receptor 2-positive metastatic breast cancer administered as first-line treatment: the M77001 study group. J Clin Oncol 23:4265-4274.

3. Fabi A, Metro G, Ferretti G, Giannarelli D, Di CS, Papaldo P, Mottolese M, Carlini P, Felici A, Russillo M, Cognetti F (2008) Do HER-2 positive metastatic breast cancer patients benefit from the use of trastuzumab beyond disease progression? A mono-institutional experience and systematic review of observational studies. Breast 17:499-505.

4. Cancello G, Montagna E, D'Agostino D, Giuliano M, Giordano A, Di LG, Plaitano M, De PS, De Laurentiis M (2008) Continuing trastuzumab beyond disease progression: outcomes analysis in patients with metastatic breast cancer. Breast Cancer Res 10:R60.

5. Extra JM, Antoine EC, Vincent-Salomon A, Delozier T, Kerbrat P, Bethune-Volters A, Guastalla JP, Spielmann M, Mauriac L, Misset JL, Serin D, Campone M, Hebert C, Remblier C, Bergougnoux L, Campana F, Namer M (2010) Efficacy of trastuzumab in routine clinical practice and after progression for metastatic breast cancer patients: the observational Hermine study. Oncologist 15:799-809.

6. Montemurro F, Redana S, Viale G, Sanna G, Donadio M, Valabrega G, del CB, Bottini A, Botti G, Paolo dei TA, Jacomuzzi ME, Di BM, Danese S, Clavarezza M, Kulka J, Di PS, Durando A, Sapino A, Aglietta M (2008) Retrospective evaluation of clinical outcomes in patients with HER2-positive advanced breast cancer progressing on trastuzumab-based therapy in the pre-lapatinib era. Clin Breast Cancer 8:436-442.

7. Von Minckwitz G, Du bois A, Schmidt M, Maass N, Cufer T, de Jongh FE, Maartense E, Zielinski C, Kaufmann M, Bauer W, Baumann KH, Clemens MR, Duerr R, Uleer C, Andersson M, Stein RC, Nekljudova V, Loibl S (2009) Trastuzumab beyond progression in human epidermal growth factor receptor 2-positive advanced breast cancer: a german breast group 26/breast international group 03-05 study. J Clin Oncol 27:1999-2006.

8. Blackwell KL, Burstein HJ, Storniolo AM, Rugo H, Sledge G, Koehler M, Ellis C, Casey M, Vukelja S, Bischoff J, Baselga J, O'Shaughnessy J (2010) Randomized study of Lapatinib alone or in combination with trastuzumab in women with ErbB2-positive, trastuzumab-refractory metastatic breast cancer. J Clin Oncol 28:1124-1130.

9. Therasse P, Arbuck SG, Eisenhauer EA, Wanders J, Kaplan RS, Rubinstein L, Verweij J, Van Glabbeke M, Van Oosterom AT, Christian MC, Gwyther SG (2000) New guidelines to evaluate the response to treatment in solid tumors. European Organization for Research and Treatment of Cancer, National Cancer Institute of the United States, National Cancer Institute of Canada. J Natl Cancer I 92:205-216.

10. Rosenbaum PR Rubin DB (1983) The central role of the propensity score in observational studies for causal effects. Biometrika 70:41-55.

11. Breiman L (2001) Random Forests. Machine Learning 45:5-32.

12. Austin PC, Grootendorst P, Anderson GM (2007) A comparison of the ability of different propensity score models to balance measured variables between treated and untreated subjects: a Monte Carlo study. Stat Med 26:734-753.

13. Clayton DG (1978) A model for association in bivariate life tables and its application in epidemiological studies of familial tendency in chronic disease incidence. Biometrika 65:141-151.

14. Ishwaran H, Kogalur UB, Blackstone EH, Lauer MS (2008) Random Survival Forests. forthcoming in Annals of Applied Statistics

15. Grambsch P Therneau T (1994) Proportional hazards tests and diagnostics based on weighted residuals. Biometrika 81:515-526.

16. Iacus SM Porro G (2007) Missing data imputation, matching and other applications of random recursive partitioning. Computational Statistics \&data Analysis 52:733-789. 
17. Montemurro F, Donadio M, Clavarezza M, Redana S, Jacomuzzi ME, Valabrega G, Danese S, Vietti-Ramus G, Durando A, Venturini M, Aglietta M (2006) Outcome of patients with HER2-positive advanced breast cancer progressing during trastuzumab-based therapy. Oncologist 11:318-324.

18. Spector NL Blackwell KL (2009) Understanding the mechanisms behind trastuzumab therapy for human epidermal growth factor receptor 2-positive breast cancer. J Clin Oncol 27:5838-5847.

19. Gennari R, Ménard S, Fagnoni F, Ponchio L, Scelsi M, Tagliabue E, Castiglioni F, Villani L, Magalotti C, Gibelli N, Oliviero B, Ballardini B, Da Prada G, Zambelli A, Costa A (2004) Pilot study of the mechanism of action of preoperative trastuzumab in patients with primary operable breast tumors overexpressing HER2. Clin Cancer Res 10:5650-5655.

20. Pandolfi PP (2004) Breast cancer - loss of PTEN predicts resistance to treatment. N Engl J Med 351:2337-2338.

21. Nahta R, Takahashi T, Ueno NT, Hung MC, Esteva FJ (2004) P27(kip1) down-regulation is associated with trastuzumab resistance in breast cancer cells. Cancer Res 64:3981-3986.

22. Scaltriti M, Rojo F, Ocana A, Anido J, Guzman M, Cortes J, Di CS, Matias-Guiu X, Cajal S, Arribas J, Baselga J (2007) Expression of p95HER2, a truncated form of the HER2 receptor, and response to anti-HER2 therapies in breast cancer. J Natl Cancer I 99:628-638.

23. Shattuck DL, Miller JK, Carraway KL, III, Sweeney C (2008) Met receptor contributes to trastuzumab resistance of Her2-overexpressing breast cancer cells. Cancer Res 68:1471-1477.

24. Mocanu MM, Fazekas Z, Petras M, Nagy P, Sebestyen Z, Isola J, Timar J, Park JW, Vereb G, Szollosi J (2005) Associations of ErbB2, beta1-integrin and lipid rafts on Herceptin (Trastuzumab) resistant and sensitive tumor cell lines. Cancer Lett 227:201-212.

25. Motoyama AB, Hynes NE, Lane HA (2002) The efficacy of ErbB receptor-targeted anticancer therapeutics is influenced by the availability of epidermal growth factor-related peptides. Cancer Res 62:3151-3158.

26. Shah C, Miller TW, Wyatt SK, McKinley ET, Olivares MG, Sanchez V, Nolting DD, Buck JR, Zhao P, Ansari MS, Baldwin RM, Gore JC, Schiff R, Arteaga CL, Manning HC (2009) Imaging biomarkers predict response to anti-HER2 (ErbB2) therapy in preclinical models of breast cancer. Clin Cancer Res 15:4712-4721. 


\section{LEGENDS}

Figure 1. CONSORT Diagram of the Italian retrospective study

Figure 2. Overall survival from the first documented progression under trastuzumab therapy for both responder (a) and non-responder groups (b) according to discontinuation (dotted) and continuation (solid line) of trastuzumab treatment.

Figure 3 Predicted survivor function from the first documented progression under trastuzumab therapy for matched patients with disease progression, according to discontinuation/continuation of trastuzumab treatment and according to response/no response: discontinuation and response (D/R, solid line), discontinuation and no response (D/NR, dashed), continuation and response (C/R, dotted), and continuation and no response (C/NR, dot-dashed).

Table 1. Descriptive statistics of the unmatched study sample and of the subgroups with suspension/continuation of Trastuzumab treatment at disease progression. Continuous covariates and counts: median and (interquartile range). Categorical covariates: absolute and (relative) frequency.

\begin{tabular}{|c|c|c|c|c|}
\hline & Total sample & $\begin{array}{c}\text { Treatment } \\
\text { continuation } \dagger\end{array}$ & $\begin{array}{c}\text { Treatment } \\
\text { suspension } \dagger\end{array}$ & $\mathbf{p}^{\S}$ \\
\hline Total & 272 & $154(56.6)$ & $118(43.4)$ & \\
\hline $\begin{array}{l}\text { Patient-related variables } \\
\text { Age (years) } \\
\text { Menopause } \\
\text { LVEF }(\%) \\
\text { Dead patients }\end{array}$ & $\begin{array}{c}54(44-61) \\
184(69.4) \\
64(59-68) \\
131(48.2)\end{array}$ & $\begin{array}{c}53(40-60) \\
99(65.1) \\
64(58-68) \\
63(40.9)\end{array}$ & $\begin{array}{c}56(48-63) \\
85(75.2) \\
63(59-67) \\
68(57.6)\end{array}$ & $\begin{array}{l}0.009 \\
0.082 \\
0.846 \\
0.007\end{array}$ \\
\hline $\begin{array}{l}\text { Tumor-related variables } \\
\text { ER positive } \\
\text { PgR positive } \\
\text { Grade (I+II vs. III) } \\
\text { Ductal histotype } \\
\text { N. of resected lymph nodes } \\
\text { N. of positive lymph nodes }\end{array}$ & $\begin{array}{c}133(49.8) \\
104(39.2) \\
160(70.8) \\
196(83.8) \\
17(12-22) \\
4(1-10)\end{array}$ & $\begin{array}{c}69(45.1) \\
52(34.4) \\
85(68.5) \\
121(83.4) \\
18(12-23) \\
4(1-9)\end{array}$ & $\begin{array}{c}64(56.1) \\
52(45.6) \\
75(73.5) \\
75(84.3) \\
15(12-20) \\
3(1-10)\end{array}$ & $\begin{array}{l}0.084 \\
0.076 \\
0.464 \\
1.000 \\
0.037 \\
0.704\end{array}$ \\
\hline $\begin{array}{l}\text { Relapses and recurrences } \\
\text { Target/not target metastases }\end{array}$ & $38(14.0)$ & $13(8.4)$ & 25 (21.2) & 0.001 \\
\hline $\begin{array}{lr} & \text { Not Visceral } \\
\text { Other } \\
\text { N. of metastatic lesions } \\
\text { Multiple metastases } \\
\text { Site of metastasis }\end{array}$ & $\begin{array}{l}62(22.8) \\
172(63.2) \\
2(1-3) \\
194(71.3) \\
30(11)\end{array}$ & $\begin{array}{l}30(19.5) \\
111(72.1) \\
2(2-3) \\
124(80.5) \\
10(6.5)\end{array}$ & $\begin{array}{l}32(27.1) \\
61(51.7) \\
2(1-3) \\
70(59.3) \\
20(16.9)\end{array}$ & $\begin{array}{c}0.010 \\
<0.001 \\
0.022\end{array}$ \\
\hline $\begin{array}{l}\text { N. of target lesions } \\
\text { Size of first metastatic lesion at } \\
\text { progression }(\mathrm{mm})\end{array}$ & $\begin{array}{c}12(4.4) \\
15(5.5) \\
215(79.0) \\
1(1-2) \\
22(15-35)\end{array}$ & $\begin{array}{c}5(3.2) \\
8(5.2) \\
131(85.1) \\
2(1-2) \\
20(11-30)\end{array}$ & $\begin{array}{c}7(5.9) \\
7(5.9) \\
84(71.2) \\
1(1-2) \\
25(20-40)\end{array}$ & $\begin{array}{l}0.001 \\
0.005\end{array}$ \\
\hline $\begin{array}{l}\text { Associated therapies } \\
\text { Taxane } \\
\text { Gemcitabine } \\
\text { Aromatase inhibitor } \\
\text { Other drugs }\end{array}$ & $\begin{array}{c}153(56.3) \\
23(8.5) \\
34(12.5) \\
23(8.5)\end{array}$ & $\begin{array}{c}106(68.8) \\
22(14.3) \\
24(15.6) \\
15(9.7)\end{array}$ & $\begin{array}{c}47(39.8) \\
1(0.8) \\
10(8.5) \\
8(6.8)\end{array}$ & $\begin{array}{c}<0.001 \\
<0.001 \\
0.079 \\
0.384\end{array}$ \\
\hline $\begin{array}{l}\text { RECIST response to first-line } \\
\text { trastuzumab-based therapy }\end{array}$ & $123(45.2)$ & $79(51.3)$ & $44(37.3)$ & 0.021 \\
\hline
\end{tabular}

$\S$ P-value of the test of independence between covariates and discontinuation/continuation of Trastuzumab treatment at progression. Continuous covariates and counts: Wilcoxon's rank-sum test statistic. Categorical covariates: Fisher's exact test. 
HER2-positive breast carcinoma patients treated with first line trastuzumab treatment for metastatic disease in 2000-2001 yr in Italian hospitals and progressed upon this first-line trastuzumab treatment

Patients with RECIST response to first-line trastuzumab treatment $n=123$ Patients without RECIST response to first-line trastuzumab treatment $n=149$
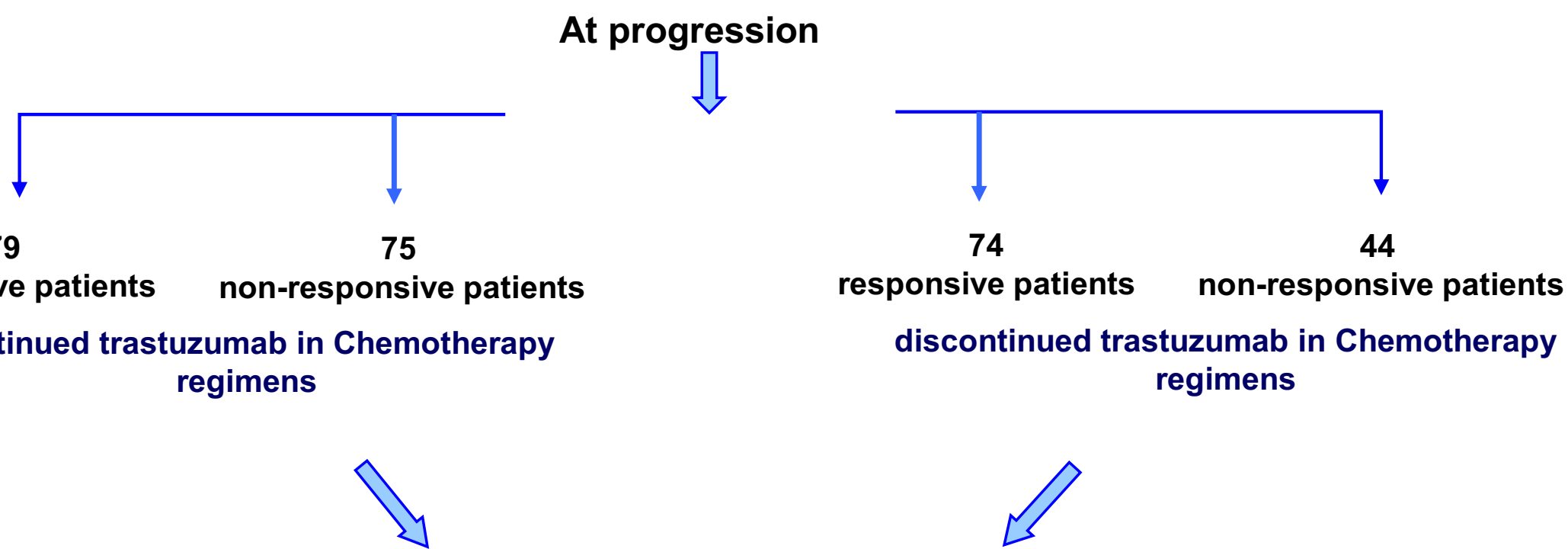

Follow-up until 2007

Analysis of the overall survival from first progression upon first line trastuzumab treatment according to continuation/discontinuation of trastuzumab at progression 
Figure

Click here to download Figure: Figure2.ppt

a

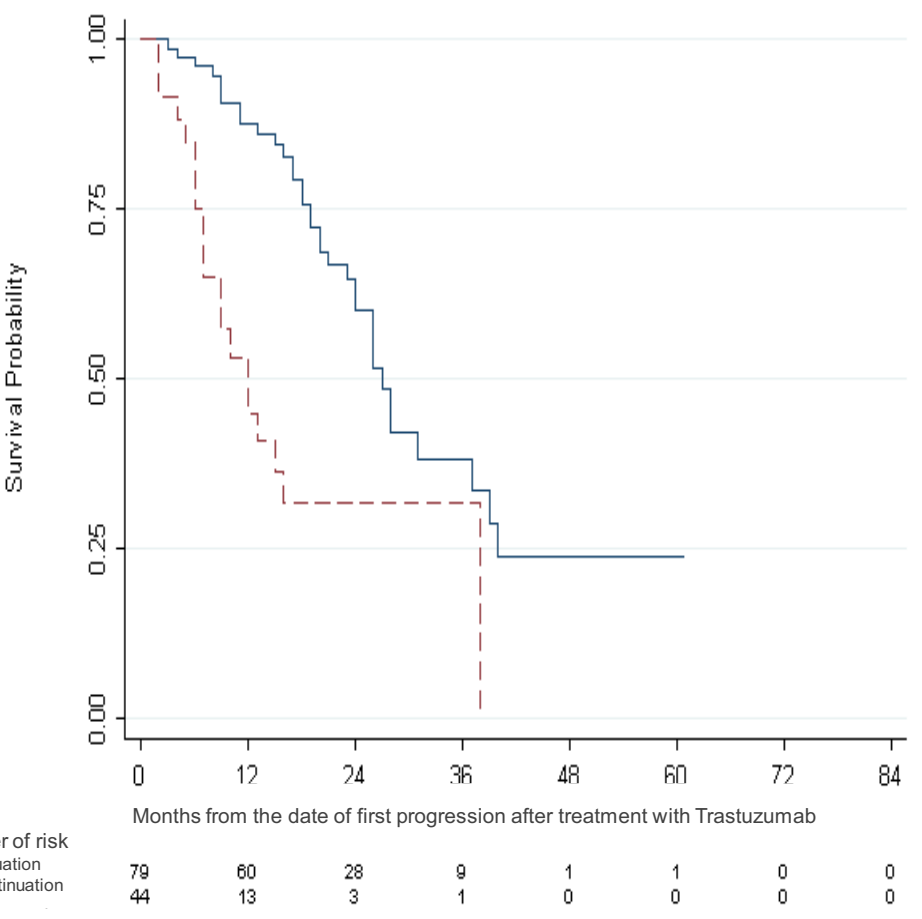

b

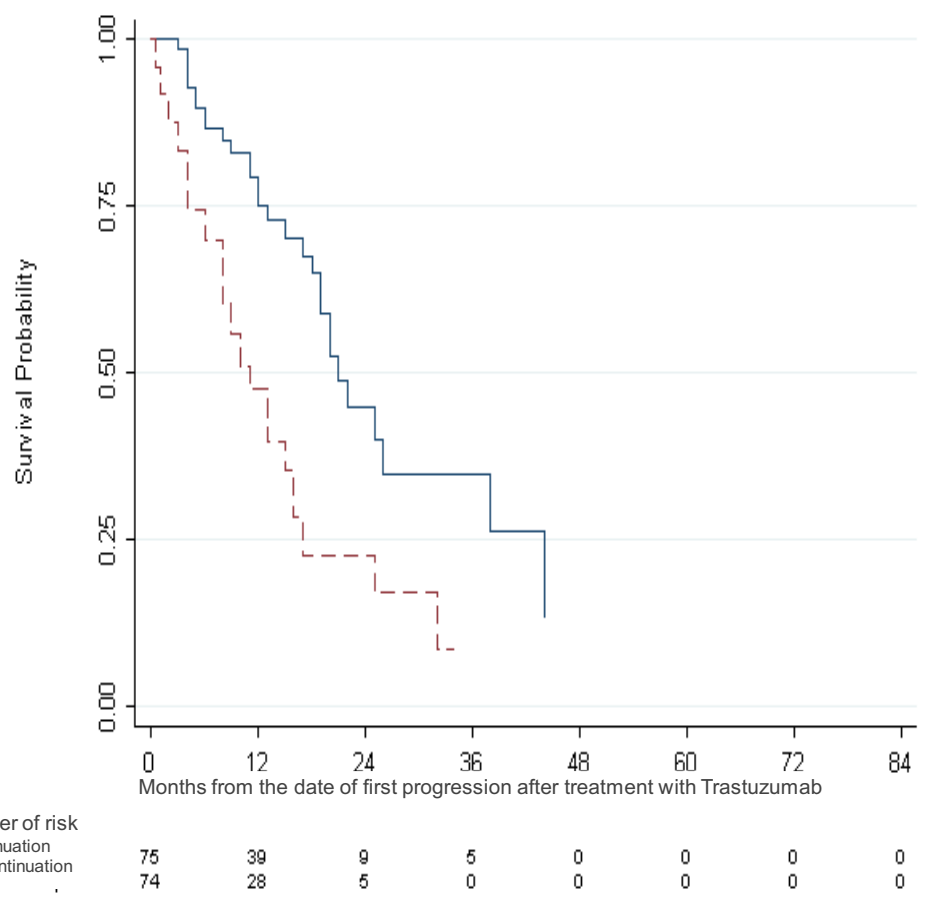


Figure

Click here to download Figure: Figure3_revised.ppt

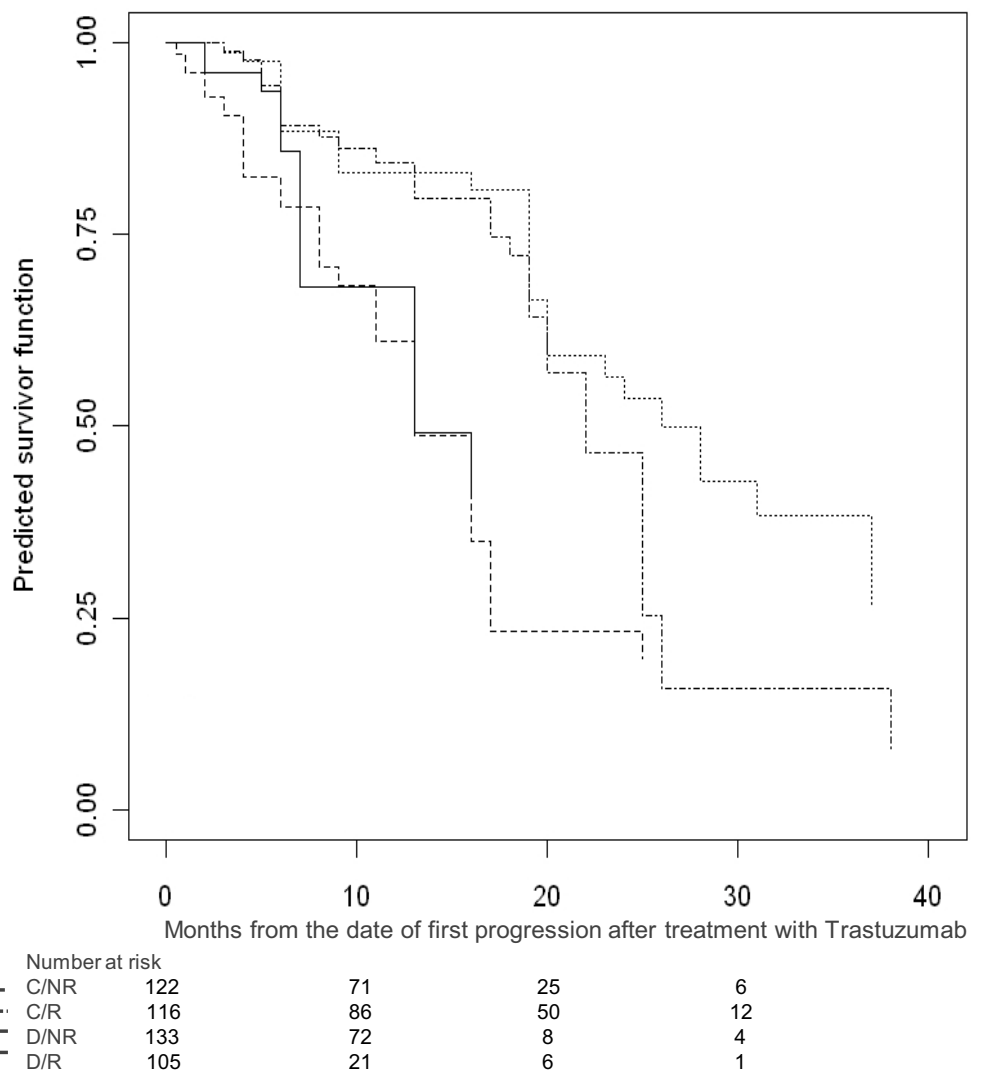

